\section{PWE-199 OUALITY OF FLEXIBLE SIGMOIDOSCOPY FOR COLORECTAL CANCER SCREENING: ARE WE THERE YET?}

doi:10.1136/gutjnl-2012-302514d.199

S K Butt, ${ }^{*}$ H Defoe, K Besherdas. Department of Gastroenterology, Chase Farm Hospital, London, UK

Introduction Colorectal cancer (CRC) screening with flexible sigmoidoscopy (FS) has been associated with reduced incidence and mortality from CRC. The NHS in UK is introducing FS screening for all men and women at 55 years of age following a multicentre randomised controlled trial which found that FS was a safe and practical test which, when offered only once conferred a substantial and lasting benefit. Quality in performance of FS will be important for success within the screening programme. One aspect of quality of FS performance is the depth of insertion. The definition of an adequately inserted screening FS is subjective and not currently defined. However, in clinical practice, one should examine as much of the distal colon as possible, recognising that it can be difficult to precisely define the furthest extent. It is expected that the rectum and sigmoid colon should be inspected completely and the descending and more proximal colon can often also be visualised. By taking the analogue of colonoscopy one could expect the "decending colon intubation rate" to be at least $90 \%$. In addition, the preparation should be such that the endoscopist is confident that lesions other than small polyps ( $5 \mathrm{~mm}$ or smaller) are not obscured. If adequate insertion is not achieved or the sigmoid colon is not adequately prepared, the procedure may have to be repeated increasing the burden on an already overstretched endoscopy service. Methods To assess the completion rate (depth of insertion in FS) defined as examination up to at least the decending colon and reasons for incompletion. A single centre, retrospective study in a district general hospital endoscopy unit in London of consecutive FS over a 2-month period in 2011. The FS reports were scrutinised for depth of insertion and if splenic/decending colon was not reached the reason for this.

Results Within the study period, the splenic flexure was reached in $40 \%$ (58 of a 148), and the decending colon in $66 \%$ (94 of 148). The reasons stated for not reaching the decending colon/splenic flexure include: inadequate bowel prep (26/148), pathology encountered $(16 / 148)$, patient discomfort (4/148), planned limited procedure (7/148). In $87 / 148$ no reason for not reaching the splenic flexure/ decending was documented.

Conclusion In this study, in the majority of patients undergoing FS the splenic flexure in not reached. Only $66 \%$ of patients have the rectum and sigmoid colon assessed during planned FS. The reasons for failure of examination to decending colon is not documented in the majority, and this may be because clear guidelines on "decending colon intubation rates" are not available. In order to implement FS as screening for CRC we recommend development of clear quality standard including one of depth of intubation (or "decending colon intubation rate") as is currently available for colonoscopy.

Competing interests None declared.

\section{PWE-200 THE IMPACT OF BOWEL PREPARATION ON OTHER COLONOSCOPY OUALITY INDICATORS}

doi:10.1136/gutjnl-2012-302514d.200

S K Butt, ${ }^{*} \mathrm{H}$ Defoe, K Besherdas. Department of Gastroenterology, Chase Farm Hospital, London, UK

Introduction Poor quality of bowel preparation may be a major obstacle to the effectiveness of colonoscopy. Poor bowel preparation results in repeat of the procedure or shortening the interval of repeat scheduled procedure all of which increase the economic burden of the test. Quality indicators of colonoscopy are an important assessment of an endoscopy unit to fulfil for formal accreditation from JAG (Joint Advisory Group of Gastrointestinal Endoscopy). These indicators include the caecal intubation rate, adenoma detection rate and polyp retrieval rate. Poor quality bowel preparation may affect all of these quality indicators of colonoscopy. The impact on the quality of bowel preparation on completion of colonoscopy and polyp detection/retrieval was assessed in this study.

Methods To test the hypothesis that poor bowel preparation results in reduced caecal intubation, reduced adenoma detection and polyp retrieval rates. This was a retrospective analysis of all colonoscopies performed at a district general hospital in the North of London for 1-year from June 2009. The quality of bowel preparation was documented as good, satisfactory or poor. The caecal intubation rate, adenoma detection rate and polyp retrieval rate within each of the types of quality of bowel preparation was scrutinised.

Results A total of 1261 colonoscopies were performed in the study period. The caecal intubation rates were $94 \%, 86 \%$ and $82 \%$ for good, satisfactory and poor bowel preparation respectively. The polyp detection rate were $20 \%, 20 \%$ and $16 \%$ and polyp retrieval rate of $92 \%, 87 \%$ and $77 \%$ for good, satisfactory and poor bowel preparation respectively.

Conclusion Patients undergoing colonoscopy whose bowel preparation is poor have significantly lower rates of caecal intubation, polyp detection and polyp retrieval compared those with good bowel preparation. This study demonstrates the importance for units to endeavour to improve bowel preparation for patients undergoing colonoscopy to improve completeness of colonoscopy. While individual colonoscopist will look to improve their quality indicators of colonoscopy by safer sedation scores and improval in withdrawal times, the quality of bowel prep needs to be assessed by the unit.

\section{Abstract PWE-200 Table 1}

\begin{tabular}{llllcccc}
\hline & & $\begin{array}{l}\text { Caecal } \\
\text { intubation }\end{array}$ & & $\begin{array}{l}\text { Polyp } \\
\text { detection }\end{array}$ & & $\begin{array}{l}\text { Polyp } \\
\text { retrieval }\end{array}$ \\
Preparation & Total & (CI) & Cl \% & (PD) & PD \% & (PR) & PR \% \\
\hline Good & 440 & 415 & 94.3 & 88 & 20 & 81 & 92 \\
Satisfactory & 661 & 573 & 86.6 & 134 & 20 & 117 & 87 \\
Poor & 184 & 152 & 82.6 & 31 & 16 & 24 & 77 \\
\hline
\end{tabular}

Competing interests None declared.

\section{PWE-201 TECHNIQUES FOR REMOVAL OF POLYPS FROM THE RIGHT COLON: HOW ARE WE DOING THEM?}

doi:10.1136/gutjnl-2012-302514d.201

S K Butt, ${ }^{*}$ K Besherdas. Department of Gastroenterology, Chase Farm Hospital, London, UK

Introduction Polypectomy is a commonly performed therapeutic intervention and all colonoscopists should be able to perform the procedure safely and effectively. Complications can include perforation and bleeding. Practice has evolved considerably in recent years and there is a range of equipment and techniques available for different clinical settings. The recommended technique for removal of polyps from the right colon include cold snaring for polyps upto $7 \mathrm{~mm}$ and hot snare for polyps $8 \mathrm{~mm}$ or greater. It is recommended to avoid cold biopsy except in tiny polyps and to avoid using hot biopsy altogether. We wanted to assess the polypectomy techniques used in our hospital and compare these to recommendations provided by the British Society of Gastroenterology guidance document. 